\title{
Total En Bloc Spondylectomy for Lumbar Renal Cell Carcinoma and Review of the Literature
}

\author{
Darweesh Al-Khawaja ${ }^{1 *}$, Tamadur Mahasneh ${ }^{2}$, Jonathan $\mathrm{Li}^{3}$, Sue-Ellen Holmes ${ }^{4}$ \\ ${ }^{1}$ Neurosurgical Department, Nepean Hospital, Kingswood, NSW, Australia \\ ${ }^{2}$ Department of Neuropathology, Royal Prince Alfred Hospital, Camperdown, NSW, Australia \\ ${ }^{3}$ Australian School of Advanced Medicine, Macquarie University, Sydney, Australia \\ ${ }^{4}$ Researcher, Private Neurosurgical Practice, Penrith, NSW, Australia \\ Email: "braindoc@tpg.com.au
}

Received November 13, 2013; revised December 13, 2013; accepted December 21, 2013

Copyright (c) 2014 Darweesh Al-Khawaja et al. This is an open access article distributed under the Creative Commons Attribution License, which permits unrestricted use, distribution, and reproduction in any medium, provided the original work is properly cited. In accordance of the Creative Commons Attribution License all Copyrights (C) 2014 are reserved for SCIRP and the owner of the intellectual property Darweesh Al-Khawaja et al. All Copyright (C) 2014 are guarded by law and by SCIRP as a guardian.

\section{ABSTRACT}

Introduction: Total en bloc spondylectomy (TES) is gaining increasing favour as a treatment of choice for cancers of the spine that are resistant to radiological and chemotherapeautic intervention such as renal cell carcinoma (RCC). Until recently, RCC of the lumbar spine has presented a surgical challenge due to anatomical and vascular constraints. The development of the combined posterior-anterior en bloc spondylectomy offers improved access to the lumbar region. This case report and review of the literature presents a combined posterior-anterior lumbar en bloc spondylectomy for RCC involving L3 vertebra, which we believe is the first reported in Australia. Methods: A 46-year-old male with a seven-year history of renal cell carcinoma resulting in a left nephrectomy presented with a lytic lesion involving the $L 3$ vertebral body, extending to the epidural space and compressing the cauda equina and left L3 and L4 nerve roots on MRI. A literature review revealed ten previous cases of the posterior-anterior TES in the lumbar spine for cancerous lesions but none from Australia. Results: A posterior-anterior TES and L2-L4 fusion was performed to remove a cancerous renal cell carcinoma of L3 with wide margins. Blood loss was the major complication. The patient remains recurrence free at nineteen months post procedure. Conclusion: Despite being an aggressive and invasive procedure, TES is rapidly becoming the treatment of choice for curative and palliative care in select patients with isolated metastatic tumours of the lumbar spine.

\section{KEYWORDS}

En Bloc Spondylectomy; Renal Cell Carcinoma; Lumbar Spine; En Bloc Spondylectomy

\section{Introduction}

Increasingly, total en bloc spondylectomy (TES) developed by Tomita et al., [1-4] where the tumour is removed in an entire encapsulated piece, has proven effective at extending the long-term survival and functional outcomes for patients with metastatic disease of the spine. A recent comprehensive review demonstrates the beneficial impact on morbidity and mortality of margin-free surgical resection [5]. This is particularly the case for isolated, solitary spinal tumours seeded from cancers such as renal cell carcinoma (RCC), which are resistant to radiation

"Corresponding author. and chemotherapeutic regimes [6,7]. At the clinically recommended dosage, radiotherapy can also compromise the integrity of surrounding neural elements [5]. Approximately $29 \%$ of RCC exhibited spinal metastases postmortem [8]. Achievement of wide marginal resection with TES is not only feasible; the technique has attained an encouraging reduction in recurrence with relatively low complications [9].

However, TES is not without controversy as a highly invasive palliative measure, demanding an advanced level of surgical proficiency [10]. This is particularly so in the lower lumbar spine due to extensive vascularisation and unique anatomy, and as such TES in this region is 
less common with fewer examples in the literature [10, 11]. In addition, the posterior only approach favoured for lumbar spondylectomy carries an increased concern for injury to the major vessels due to indirect visualisation of ventral structures [5]. This case report and review of the literature presents a combined posterior-anterior lumbar en bloc spondylectomy for RCC involving L3 vertebra, which we believe is the first reported in Australia.

\section{Case Report}

\subsection{History}

Our patient is 46-year-old male with a history of RCC diagnosed seven years prior resulting in a left nephrectomy. He presented with lower back pain, parasthesia and radiculopathy in the L3 distribution down his left leg after twisting his back at work.

\subsection{Examination}

On Examination the patient presented with tenderness of the midlumbar region and weak left knee extention of power $4 / 5$ with diminished left knee jerk. He had decreased sensation around left L3, L4 dermatomes.

\subsection{Imaging}

An MRI of the lumbar spine revealed a lytic lesion involving the L3 vertebral body, extending to the epidural space and compressing the cauda equina and left L3 and L4 nerve roots (Figures 1 and 2). Surprisingly, a bone SPECT scan returned as negative for any significant hot spots.

\subsection{Treatment}

A revised Tokuhashi score of 10 (with 9 being the recommended cut-off for alternative palliative measures), in

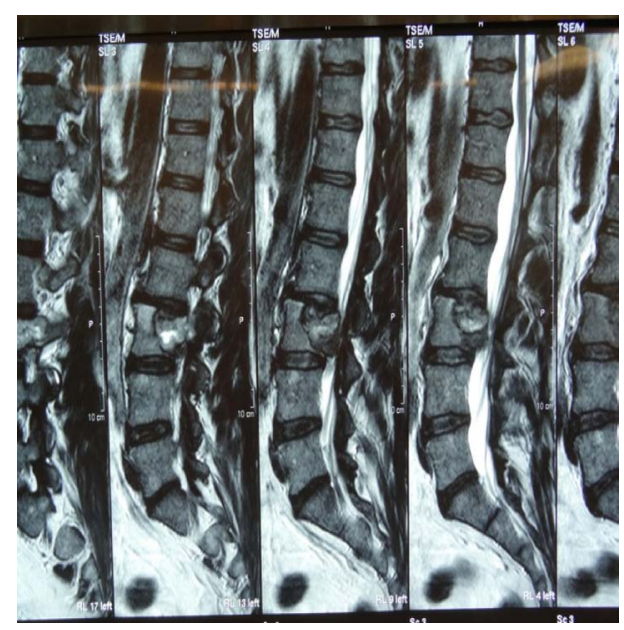

Figure 1. Pre-Operative T2 weighted sagittal MRI of L3 RCC tumour.

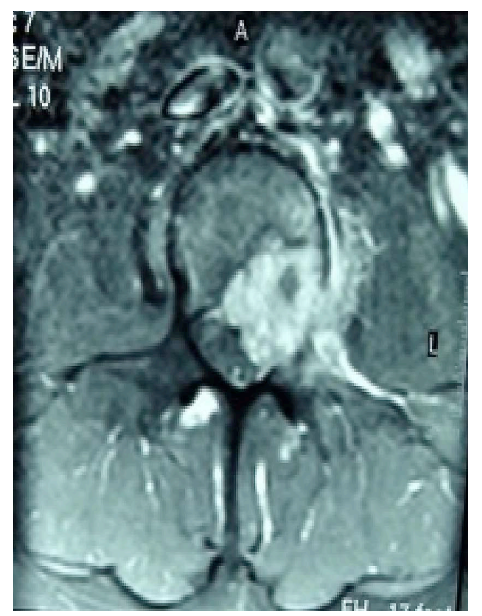

Figure 2. Pre-Operative T2 weighed axial MRI of L3 RCC tumour.

conjunction with multi-disciplinary team review, achieved consensus on his eligibility for the En bloc Spondylectomy procedure.

Embolisation of lumbar arteries feeding the tumour occurred one day prior to admission for surgery.

\subsection{Operation}

\subsubsection{Posterior Approach}

The patient was placed in a prone position on the Jackson table. After a midline incision, a bilateral periosteal dissection was completed to completely expose the left L3/4 facet joint and transverse process (TP) on both sides. Applying the Gigli saw from beneath the left TP, the left L3 pedicle and TP were cut in a superior posterior direction. The right L3 lamina was removed.

On the left side, the L3 vertebral body was dissected from the psoas muscle to the anterior border, L2 and L4 pedicle screws inserted and connected with rods and cross link. Using an osteotome a sagittal split was completed on the left side $5 \mathrm{~mm}$ from the border of the tumor, then advanced anteriorly to the anterior cortical edge of the vertebral body. The tumor was dissected from the dura completely and epidural veins were coagulated. Every effort was made to avoid breaching the tumour and to keep the capsule intact (Figure 3). L2/3 and L3/4 discectomy to the level of the sagittal split on the right side and as far as possible both anteriorly and laterally on the left side occurred, before removal of the annulus on the left side, and finally closure.

\subsubsection{Anterior Approach}

The patient was rotated $180^{\circ}$. A midline abdominal incision was performed from the retroperitoneal approach, whereby the Aorta was mobilized. The L3 vertebral body with the above and below disc spaces were identified and an L2/3 and L3/4 discectomy completed from the front. 


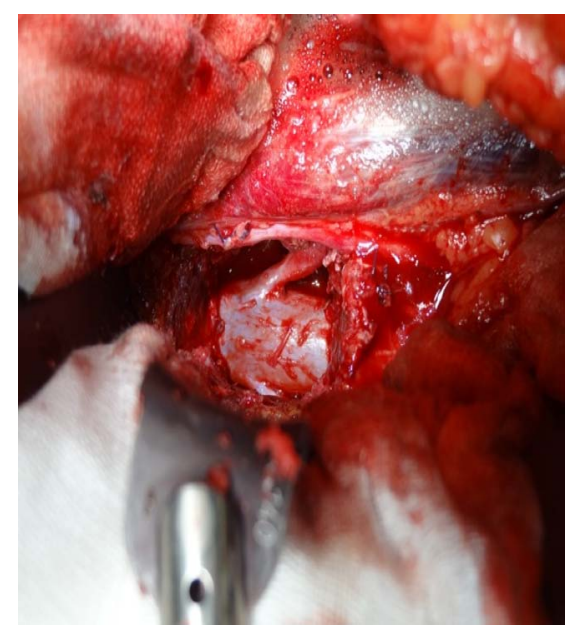

Figure 3. Anterior view of the dural sac and left L3 nerve root after $L 3$ vertebra removed.

L3 vetebrectomy removed the tumour anteriorly (Figure 4) with subsequent interbody fusion using an expandable cage with bone graft and bone marrow aspirate.

\subsubsection{Postoperative Course}

Transient weakness (3/5 power) of left sided hip flexion was likely due to dissection of the psoas muscle. The patient required a blood transfusion and developed deep vein thrombosis of both lower limbs for which he received a prolonged course of anti-coagulants. Mobilisation was encouraged after three days with a thoracolumber corset worn. Post-operative management involved adjuvant radiotherapy. A postoperative CT scan showed screws and cage in good position (Figure 5).

\subsubsection{Histopathology}

Histopathology revealed a low-grade clear cell renal carcinoma confined within the vertebral body with wide free margins (Figure 6).

\subsubsection{Follow-Up}

On eighteen months follow-up the patient remained free of local recurrence.

\section{Discussion}

Historically, radiotherapy has been the treatment of choice for spinal metastases because surgery carried the risk of substantial morbidity due to tumour cell spillage at the resection site and recurrence due to residual cancerous tissue [11]. Applying Enneking et al.'s [12] concept of tumour compartmentalisation due to anatomic barriers in the spine, the anterior longitudinal ligament, cartilaginous endplate and annulus fibrosus contain the progression of tumour spread to the vertebra and serve to retard distant proliferation [2-4,11]. Since the introduction of TES in 1989 [2] this surgical intervention has in-

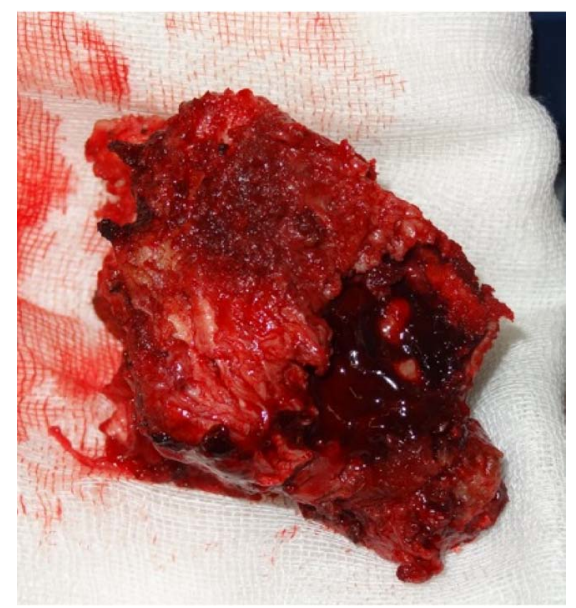

Figure 4. L3 vertebra and en bloc tumour excision.

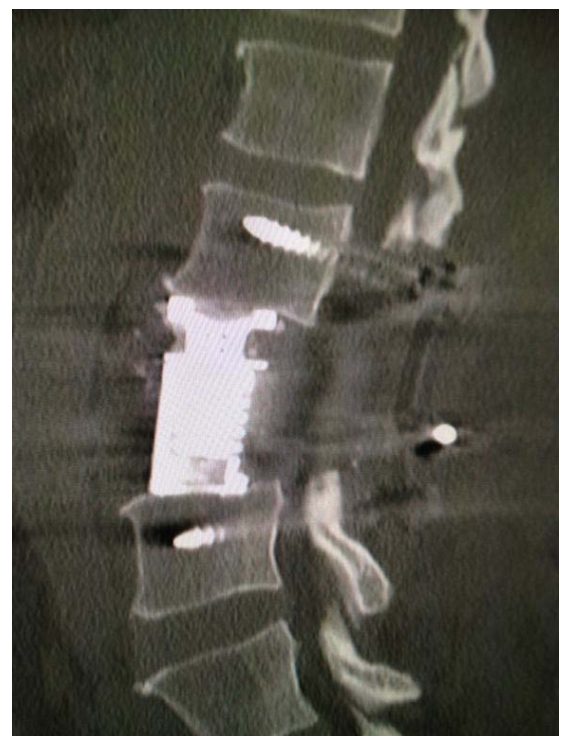

Figure 5. Post-operative sagittal CT of L3 corpectomy.

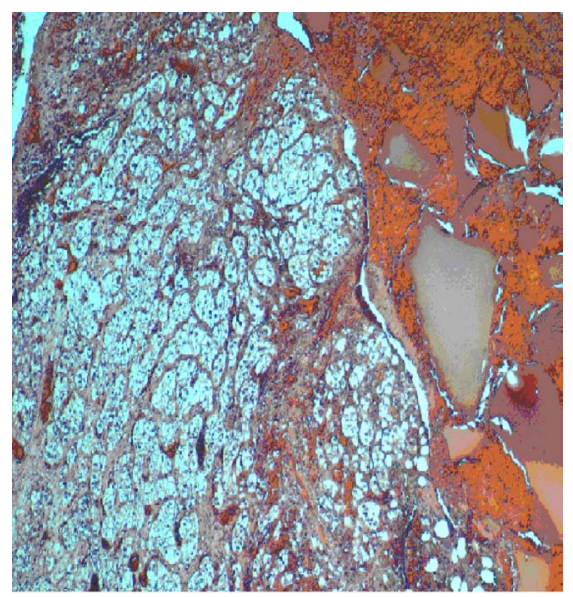

Figure 6. Histopathology of clear cell type renal carcinoma (Haematoxylin and Eosin, original magnification $\times 400$ ). Extensive replacement of bony tissue by nests of malignant cells with abundant clear cytoplasm and small round nuclei. 
creasingly enhanced the quality of life and improved survival chances for all oncology patients with the exception of those suffering complete paraplegia [13]. Furthermore, a recent randomised trial comparing radiotherapy with surgery for spinal cord decompression in metastatic cancer, where the endpoint measured was reestablishment of amubulation, was halted early due to the overwhelming superiority of surgical treatment [14].

Life expectancy has been shown to be the best predictor of prognostic optimism for patients with spinal metastases $[15,16]$. The Revised Tokuhashi Scale assesses a patient's eligibility for TES on the basis of life expectancy across six oncological domains: general performance status, number of extraspinal bone metastases foci, number of metastases in the vertebral body, metastases to the major internal organs, primary site of cancer, and the presence of palsy. The scale provides an evidence-based rationale to informing the surgeon's decision to treat spinal metastases with radical surgery [15]. In this instance, the RCC patient was under 65 years-old, achieved a good performance status, and had a solitary, intracompartmental tumour with no sign of systemic disease, and thus was considered favourable for therapeutic intervention and post-operative longevity [10,15].

Due to the poor response of RCC to radiation and chemotherapy, approximately $50 \%$ of patients die within the first year of presentation with only $10 \%$ surviving more than five years $[10,13]$. In their recent comprehensive review of TES, Cloyd and colleagues (2010) reported an average five year disease-free survival of 77 patients with solitary metastatic tumours of the thoracic and lumbar spine, including 28 cases of RCC, ranging from approximately $25 \%$ - 56\%. A mean time to recurrence of 26 months was also found [5]. A more conservative figure for five year survival rates in patients undergoing TES for spine metastases of greater than $15 \%$ by Yao et al. (2003) [17] still represents an improvement on adjuvant therapies alone. The patient herein is alive and recurrence free nineteen months post-operatively, which is consistent with the surgical goals of oncological control, spinal stabilisation, mitigation of neurological symptoms and histological diagnosis [13].

The posterior surgical approach for primary metastatic tumours of the lumbar spine is prevalent in the case series literature with an initial total of 26 patients [5]. A disadvantage of the posterior only method is the risk to major vessels due to poor visualisation of ventral structures [1,15]. As well, in an investigation of eight patients who underwent TES for solitary spinal metastases, Abe et al. (2001) linked the posterior alone approach to a $25 \%$ local recurrence for tumours anterior to the vertebral body [18].

Since, a further case-series of ten has been reported using posterior-anterior TES in the lumbar spine [10].
This approach reduces the likelihood of vascular complications and potential compromise of lumbosacral plexus nerves at the expense of longer operative times [5] due to the need to turn the patient mid-surgery. The advantage of TES for the prevention of neoplastic dissemination is particularly relevant in the highly vascular lumbar region.

In addition, wide tumour-free margins correlate most favourably with lack of local recurrence and extended patient survival $[10,15]$. In this instance, the patient had an expansile bony tumour of approximately 3 centimetres in the superior/inferior dimension on the left of the L3 vertebra, extending centrally 4.2 centimetres in the anterior-posterior and 3.3 centimeters in the transverse dimensions, involving the extra dural space, encroachment on the left pedicle and posterior pedicular elements. Wide tumour free margins were confirmed histopathologically. No relationship between bisection at a tumour afflicted pedicle and local recurrence has been found [18].

To date, none of the reported TES cases in the literature were performed in Australia. To our knowledge this is the first record of TES for lumbar RCC using the posterior-anterior approach in this country. Operating times, blood loss and complications with this procedure are high and necessitate careful consideration of the benefits for individual candidates. Despite a fifteen hour operating time and the need for a blood transfusion, the patient tolerated the procedure well and recovered full mobility within days. Such considerations though, should improve over time with application of the technique, [19] which offers a worthwhile addition to options for better outcomes and quality of life in RCC spinal tumour patients.

\section{Conclusion}

Despite being an aggressive and invasive procedure, TES is rapidly becoming the treatment of choice for curative and palliative care in select patients with isolated metastatic tumours of the spine. Ameliorating the functional impairment and resistance to radiation of RCC in the spine means it is an ideal condition for this treatment.

\section{REFERENCES}

[1] Tomita, K., Kawahara, N., Baba, H., Tsuchiya, H., Fujita, T. and Toribatake, Y. (1997) Total en bloc spondylectomy. A new surgical technique for primary malignant vertebral tumors. Spine, 22, 324-333. http://dx.doi.org/10.1097/00007632-199702010-00018

[2] Tomita, K., Kawahara, N., Baba, H., Tsuchiya, H., Nagata, S., Toribatake, Y. (1994) Total en bloc spondylectomy for solitary spinal metastases. International Orthopaedics, 18, 291-298. http://dx.doi.org/10.1007/BF00180229

[3] Tomita, K., Kawahara, N., Kobayashi, T., Yoshida, A., Murakami, H. and Akamaru, T. (2001) Surgical strategy for spinal metastases. Spine, 26, 298-306. 
http://dx.doi.org/10.1097/00007632-200102010-00016

[4] Tomita, K., Toribatake, Y., Kawahara, N., Ohnari, H. and Kose, H. (1994) Total en bloc spondylectomy and circumspinal decompression for solitary spinal metastasis. Paraplegia, 32, 36-46. http://dx.doi.org/10.1038/sc.1994.7

[5] Cloyd, J.M., Acosta, F.L., Polley, M.Y. and Ames, C.P. (2010) En bloc resection for primary and metastatic tumors of the spine: A systematic review of the literature. Neurosurgery, 67, 435-445.

[6] Fottner, A., Szalantzy, M., Wirthmann, L., Stähler, M., Baur-Melnyk, A., Jansson, V. and Roland Dürr, H. (2010) Bone metastases from renal cell carcinoma: Patient survival after surgical treatment. BMC Musculoskeletal Disorders, 11, 145-151. http://dx.doi.org/10.1186/1471-2474-11-145

[7] Jung, S.T., Ghert, M.A., Harrelson, J.M. and Scully, S.P. (2003) Treatment of osseous metastases in patients with renal cell carcinoma. Clinical Orthopaedics and Related Research, 409, 223-231.

http://dx.doi.org/10.1097/01.blo.0000059580.08469.3e

[8] Mut, M., Schiff, D. and Shaffrey, M. (2005) Metastasis to nervous system: Spinal epidural and intramedullary metastases. Journal of Neuro-Oncology, 75, 43-56. http://dx.doi.org/10.1007/s11060-004-8097-2

[9] Garcia-Picazo, A., Capilla, R.P., Pulido, R.P., Garcia de Sola, R. (1990) Utility of surgery in the treatment of epidural vertebral metastases. Acta Neurochirurgica, 103, 131-138. http://dx.doi.org/10.1007/BF01407520

[10] Melcher, I., Disch, A.C., Khodadadyan-Klostermann, S.T., Smolny, M., Stockle, U., Haas, N.P., et al. (2007) Primary malignant bone tumors and solitary metastases of the thoracolumbar spine: Result by management with total en bloc spondylectomy. European Spine Journal, 16, 11931202. http://dx.doi.org/10.1007/s00586-006-0295-5

[11] Disch, A., Druschel, C., Melcher, I., Luzzati, A. and Shaser, K. (2011) En-bloc spondylectomy for thoracolumbar primary tumours and solitary metastases of the spine. Argospine News \& Journal, 23, 163-170. http://dx.doi.org/10.1007/s12240-011-0030-x
[12] Enneking, W.F., Spanier, S.S. and Goodmann, M.A. (1980) A system for the surgical staging of musculoskeletal sarcoma. Clinical Orthopaedics, 153, 106-120.

[13] Fuchs, B., Trousdale, R.T. and Rock, M.G. (2005) Solitary bony matastasis from renal cell carcinoma: significance of surgical treatment. Clinical Orthopaedics and Related Research, 431, 187-192. http://dx.doi.org/10.1097/01.blo.0000149820.65137.b4

[14] Patchell, R.A., Tibbs, P.A., Regine, W.F., Payne, R., Saris, S., Kryscio, R.J., Mohiuddin, M. and Young, B. (2005) Direct decompressive surgical resection in the treatment of spinal cord compression caused by metastatic cancer: A randomised trial. The Lancet, 366, 643-648. http://dx.doi.org/10.1016/S0140-6736(05)66954-1

[15] Tokuhashi, Y., Matsuzaki, H., Oda, H., Oshima, M. and Junnosuke, R.A. (2005) Revised scoring system for preoperative evaluation of metastatic spine tumor prognosis. Spine, 30, 2186-2191. http://dx.doi.org/10.1097/01.brs.0000180401.06919.a5

[16] Mollahoseini, R., Farhan, F., Khajoo, A., Jouibari, M.A.M. and Gholipour, F. (2011) Is Tokuhashi score suitable for evaluation of life expectancy before surgery in Iranian patients with spinal metastases? Journal of Research in $\mathrm{Me}$ dical Sciences, 16, 1183-1188.

[17] Yao, K.C., Boriani, S., Gokaslan, Z.L. and Sundaresan, N. (2003) En bloc spondylectomy for spinal metastases: A review of the techniques. Neurosurgical Focus, 15, 1-5. http://dx.doi.org/10.3171/foc.2003.15.5.6

[18] Abe, E., Kobayashi, T., Murai, H., Suzuki, T., Chiba, M. and Okuyama, K. (2001) Total spondylectomy for primary malignant, aggressive benign, and solitary metastatic bone tumors of the thoracolumbar spine. Journal of Spinal Disorders, 14, 237-246. http://dx.doi.org/10.1097/00002517-200106000-00009

[19] Tomita, K., Kawahara, N., Murakami, H. and Demura, S. (2006) Total en bloc spondylectomy for spinal tumours: Improvement of the technique and its associated basic background. Journal of Orthopaedic Science, 11, 3-12. http://dx.doi.org/10.1007/s00776-005-0964-y 